\title{
29. On Pseudo-compact Spaces and Convergence of Sequences of Continuous Functions
}

\author{
By R. W. BAGLEY \\ Mississippi Southern College, Hattisburg, Mississippi, U.S.A. \\ (Comm. by K. KunUGi, M.J.A., March 12, 1960)
}

Among the results of this paper are some generalizations of results by Iséki [4] and Isiwata [5] concerning convergence properties for sequences of real-valued continuous functions. In particular it is shown that an arbitrary topological space $X$ is pseudo-compact if and only if every sequence of continuous, real-valued functions which converges in some admissible (jointly continuous) topology for $C(X)$ converges uniformly. An additional characterization of pseudo-compact spaces is the following: If $X$ satisfies the first axiom of countability, then $X$ is pseudo-compact if and only if every sequence which converges in the compact-open topology on $C(X)$ converges uniformly.

We define several types of convergence for sequences of functions in $C(X)$, the ring: of continuous, real-valued functions on an arbitrary topological space $X$.

(a) $\left\{f_{n}\right\}$ converges to $f$ uniformly at each point of $X\left[f_{n} \rightarrow f(U P)\right]$ if, for each $x \in X$ and $\varepsilon>0$ there is an integer $N$ and a neighborhood $U_{x}$ of $x$ such that $\left|f_{n}(y)-f(y)\right|<\varepsilon$, whenever $y \in U_{x}$ and $n \geq N$.

(b) $\left\{f_{n}\right\}$ converges locally uniformly $\left[f_{n} \rightarrow f(L U)\right]$ if, for each $x \in X$, there is a neighborhood of $x$ on which $\left\{f_{n}\right\}$ converges to $f$ uniformly.

(c) $\left\{f_{n}\right\}$ converges strictly continuously to $f\left[f_{n} \rightarrow f(S C)\right]$ if, whenever $f\left(x_{n}\right)$ converges, $f_{n}\left(x_{n}\right)$ converges to the same limit. (See $[4]$.)

(d) $\left\{f_{n}\right\}$ converges jointly to $f\left[f_{n} \rightarrow f(J)\right]$ if $\left\{f_{n}\right\}$ converges to $f$ in some admissible topology for $C(X)$, i.e. a topology in which $(f, x) \rightarrow f(x)$ is a continuous mapping of $C(X) \times X$ into the real numbers. (See [5].)

In this paper we consider only continuous real-valued functions on a topological space $X$ and by convergence we mean convergence to an element of $C(X)$.

THEOREM 1. In any topological space $X$ the following are equivalent.

(i) $X$ is pseudo-compact.

(ii) Every sequence of continuous functions which converges uniformly at each point of $X$ converges uniformly.

(iii) Every sequence of continuous functions which converges locally uniformly converges uniformly. 
(iv) Every sequence of continuous functions which converges strictly continuously converges uniformly.

(v) Every equicontinuous sequence which converges pointwise converges uniformly.

(vi) Every jointly convergent sequence of continuous functions converges uniformly.

Proof. The equivalence of (i), (ii) and (iii) for arbitrary topological spaces was established in [1]. To complete the proof of the equivalence of the six properties we prove that (ii) implies (iv), (iv) implies (i), (ii) is equivalent to (v), (vi) implies (i) and (ii) implies (vi).

Proof that (ii) implies (iv). It is sufficient to show that $f_{n} \rightarrow f(S C)$ implies $f_{n} \rightarrow f(U P)$. Assume that $\left\{f_{n}\right\}$ does not converge uniformly at some point $x \in X$. Then, for some positive number $\varepsilon$, there is a sequence of points $\left\{x_{n}\right\}$ and a subsequence $\left\{f_{i_{n}}\right\}$ such that $x_{n} \in f^{-1}((f(x)-1 / n$, $f(x)+1 / n)$ ) and $\left|f_{i_{n}}\left(x_{n}\right)-f\left(x_{n}\right)\right|>\varepsilon$, where $i_{n} \geq n$. Now $f_{i_{n}} \rightarrow f(S C)$ since $\left\{f_{i_{n}}\right\}$ is a subsequence of $\left\{f_{n}\right\}$ and a subsequence of a strictly continuously convergent sequence is strictly continuously convergent. Thus, since $\left\{f\left(x_{n}\right)\right\}$ converges to $f(x),\left\{f_{i_{n}}\left(x_{n}\right)\right\}$ converges to $f(x)$ which contradicts the above sequence of inequalities.

Proof that (iv) implies (i). Assume that there is an unbounded function $g$ in $C(X)$. Define $g_{n}(x)=g(x)$ when $g(x) \leq n$ and $g_{n}(x)=n$ otherwise. Clearly $g_{n} \rightarrow g(S C)$ but $\left\{g_{n}\right\}$ does not converge uniformly.

Proof that (ii) is equivalent to (v). Suppose that $\left\{f_{n}\right\}$ is equicontinuous and converges to $f$. Let $x \in X$. If $\varepsilon>0$, by the equicontinuity, there is a neighborhood $V_{x}$ such that $\left|f_{n}(y)-f_{n}(x)\right|<\varepsilon / 3$ for each $y \in V_{x}$. By the convergence at $x$, there is a positive integer $N$ such that $\left|f_{n}(x)-f(x)\right|<\varepsilon / 3$, whenever $n \geq N$. Since $f$ is continuous there is a neighborhood $W$ such that $|f(y)-f(x)|<\varepsilon / 3$, whenever $y \in W_{x}$. Let $U_{x}=V_{x} \cap W_{x}$. If $y \in U_{x}$ and $n \geq N$, then $\left|f_{n}(y)-f(y)\right| \leq\left|f_{n}(y)-f_{n}(x)\right|$ $+\left|f_{n}(x)-f(x)\right|+|f(x)-f(y)|<\varepsilon$. Hence $f_{n} \rightarrow f(U P)$ and (ii) implies (v). For the reverse implication suppose that $f \rightarrow f(U P)$. Then, if $\varepsilon>0$ and $x \in X$ there is a positive integer $N$ and a neighborhood $U$ of $X$ such that $\left|f_{n}(y)-f_{N}(y)\right|<\varepsilon / 3$, whenever $y \in U_{0}$ and $n \geq N$. For each $i=1,2, \cdots, N$ there is a neighborhood $U$ of $x$ such that $\left|f_{i}(y)-f_{i}(x)\right|<\varepsilon / 3$, for each $y \in U_{i}$. Let $U=\bigcap_{i=0}^{N} U_{i}$. If $y \in U$, then $\left|f_{n}(y)-f_{n}(x)\right|<\varepsilon$ for $n \leq N$ and $\left|f_{n}(y)-f_{n}(x)\right| \leq\left|f_{n}(y)-f_{N}(y)\right|+\left|f_{N}(y)-f_{N}(x)\right|+\left|f_{N}(x)-f_{n}(x)\right|<\varepsilon$, whenever $n \geq N$. This completes the proof of the equivalence of (ii) and (v).

Proof that (vi) implies (i). If $g$ is in $C(X)$ and is unbounded, then defining $g_{n}(x)$ as above we see that $g_{n} \rightarrow g$ (jointly) since $\left\{g_{n}\right\}$ converges to $g$ in $C(X)$ with the admissible topology which has as a subbasis sets of the form $V(\epsilon, f,(a, b))=\{g \in C(X)|| g(x)-f(x) \mid<\varepsilon$ for each $\left.x \in f^{-1}(a, b)\right\}$ where $\epsilon, f$ and $(a, b)$ are fixed, $\epsilon>0, f \in C(X)$ and $(a, b)$ is an open interval of real numbers. To prove this let $\varepsilon>0$ and $(a, b)$ be 
any interval of real numbers. Pick $N>b+\varepsilon$. Then $g_{n}(x)=g(x)$ for each $x \in g^{-1}(a-\varepsilon, b+\varepsilon)$ and $n \geq N$. If $g \in V(\varepsilon, f,(a, b))$, then $g_{n} \in V(\in, f,(a, b))$ for $n \geq N$.

Proof that (ii) implies (vi). To prove this we show that, if $f_{n} \rightarrow f(J)$, then $f_{n} \rightarrow f(U P)$. Assume that $f_{n} \rightarrow f(J)$. Choose any point $x \in X$ and any positive number $\varepsilon$. Since $(f, x) \rightarrow f(x)$ is a continuous mapping, there is an open set $V_{f} \otimes U_{x}$ in $C(X) \otimes X$ such that $g(y) \in(f(x)$ $-\varepsilon / 3, f(x)+\varepsilon / 3)$, whenever $(g, y) \in V_{f} \otimes U_{x}$. Since $f_{n} \rightarrow f(J)$, there is a posivive integer $N$ such that $f_{n} \in V_{f}$ for $n \geq N$. Now, if $y \in U_{x}$ and $n \geq N$, then $f_{n}(y), f_{n}(x), f(y), f(x) \in(f(x)-\varepsilon / 3, f(x)+\varepsilon / 3)$. Hence $\mid f_{n}(y)$ $-f(y) \mid<\varepsilon$. This completes the proof of Theorem 1 .

LEMMA. A topological space $X$ is pseudo-compact if every sequence which converges in the compact-open topology for $C(X)$ converges uniformly.

Proof. If $g \in C(X)$ is unbounded, we define $g$ as before. (See the proof that (iv) implies (i) of Theorem 1.) Let $A$ be any compact subset of $X$ and $V$ be any open set of real numbers such that $g(A) \subset U$. Since $g(A)$ is compact, there is a real number $r \in V$ such that $g(x) \leq r$ for $x \in A$. Take $N>r$. Now $g(x)=g(x)$ for $x \in A$ and $n \geq N$. Thus $g$ is in the open set $\{f \in C(X) \mid f(A) \subset V\}$ for all $n \geq N$. This shows that $g$ converges to $g$ in the compact-open topology but not uniformly, completing the proof of the lemma.

Convergence in the compact-open topology clearly implies continuous convergence since, for a convergent sequence, the value set plus the limit is a compact set (cf. [3, p. 241]). If the topological space $X$ satisfies the first axiom of countability and $\left\{f_{n}\right\} \in C(X)$ converges to $f$ continuously, then $\left\{f_{n}\right\}$ converges uniformly at each point of $X$. To prove this let $x$ be a point with basis $V_{1}, V_{2}, \cdots, V_{n}, \cdots$ and assume that $\left\{f_{n}\right\}$ does not converge uniformly at $x$. Then for some positive number $\epsilon$ and each set $U_{n}=\bigcap_{i=1}^{n} V_{i}$ there is an integer $i_{n}>n$ and a point $x_{n} \in U_{n}$ such that $\left|f_{i_{n}}\left(x_{n}\right)-f\left(x_{n}\right)\right|>\epsilon$. Since $x_{n} \rightarrow x$ and $\left\{f_{n}\right\}$ converges continuously $f_{i_{n}}\left(x_{n}\right) \rightarrow f(x)$. This contradicts the inequalities above and consequently contradicts the assumption that $\left\{f_{n}\right\}$ does not converge uniformly at $x$.

A point $x$ is a $\mathrm{P}$-point if the interior of every countable intersection of open sets containing $x$ contains $x$. If $x$ is a P-point and $\left\{f_{n}\right\}$ converges pointwise to $f$, then, clearly $\left\{f_{n}\right\}$ converges to $f$ uniformly at $x$. Using the above results and Glicksberg's result that, in a regular pseudo-compact, every $G_{\delta}$ point has a countable basis $[2$, p. 378], we have the following.

THEOREM 2. If $X$ is a regular topological space in which every non $P$-point is a $G_{\delta}$ point, then the following are equivalent to the properties (i) through (vi) of Theorem 1. 
(vii) Every sequence of continuous functions which converges continuously converges uniformly.

(viii) Every sequence of continuous functions which converges in the compact-open topology for $C(X)$ converges uniformly.

Note that the regularity condition of Theorem 2 can be removed if " $G_{\delta}$ " is replaced by " 1 st countable".

Let $T$ be the Tychonoff plank minus the point $(\omega, \Omega)$. Since $T$ is pseudo-compact and only the points $(n, \Omega)$ fail to have a countable basis, continuous convergence implies uniform convergence; however, $T$ is not countably compact. It is not known whether or not a completely regular pseudo-compact space which satisfies the 1st axiom of countability is countably compact. Of course, countable compactness is equivalent to countable paracompactness in such a space [1].

\section{References}

[1] R. W. Bagley, E. H. Connell, and J. D. McKnight: On properties characterizing pseudo-compact spaces, Proc. Amer. Math. Soc., 9, 500-506 (1958).

[2] I. L. Glicksberg: Stone-Čech compactifications of products, Trans. Amer. Math. Soc., 90, 369-382 (1959).

[3] J. L. Kelley: General Topology, Van Nostrand (1955).

[4] K. Iséki: Pseudo-compactness and strictly continuous convergence, Proc. Japan Acad., 33, 424-428 (1957).

[5] T. Isiwata: On strictly continuous convergence of continuous functions, Proc. Japan Acad., 34, 82-86 (1958). 